\title{
A Case for Understanding User Experience Challenges confronting Indigenous Knowledge Recorders in Rural Communities in South Africa
}

\author{
Gugu KHALALA ${ }^{1}$, Ishmael MAKITLA ${ }^{2}$, Adele BOTHA ${ }^{3}$, Ronell ALBERTS ${ }^{4}$ \\ ${ }_{1,2,3,4}$ CSIR Meraka Institute, PO BOX 395, Pretoria, 0001, South Africa \\ Tel: +27 12841 2073, Fax: + 2712841 2020, \\ ${ }^{1,3}$ University of South Africa, PO BOX 392, Pretoria, 0003, South Africa \\ School of Computing \\ Tel: +27 11670 9186, Fax: + +27116709186 \\ Email: \{gkhalala, imakitla, abotha, ralberts\}@csir.co.za
}

\begin{abstract}
For centuries, rural communities have relied on their indigenous knowledge for survival and sustenance. Indigenous Knowledge (IK) is understood as local knowledge, which is unique and traditional to a specific community. Due to the fact that IK plays an important role in the general well-being of a community, IK needs to be properly collected and protected. In view of this, the South African government undertook the development of a National Recordal System (NRS). However, User Experience (UX) in IKS has been largely ignored and as a result very little is understood of UX related challenges within the IKS field. This paper makes a case for the understanding of UX challenges confronting IK Recorders in rural communities in South Africa. Understanding UX challenges will improve on the overall quality of the captured IK and is important for the enhancement of the NRS processes, tools and the system that has been deployed.
\end{abstract}

Keywords: Indigenous Knowledge, IK Recorders, User Experience

\section{Introduction}

Rural communities have over the years gained Indigenous Knowledge (IK) that has helped them to survive; sustain their lives and their environment for future generations [1]. Most of rural societies are faced with poverty and are underdeveloped and for this reason, they often find other means to sustain a living such as agriculture, cultural activities, etc. Rural people have indigenous knowledge such as agriculture, health care, food preparation, education, natural-resource management, arts and so forth. They know how to grow plants and when to harvest, which plants can be used for medicines and which plants are dangerous and poisonous, they know how to cure diseases and which plants are used for medicinal purposes and which ones are used for food [2],[3].

For a culture to survive, its folklores, legends, customs, rituals and the knowledge underpinning these must be preserved for next generations. This makes the preservation of indigenous knowledge an absolute necessity for the preservation of a people and their identity, which is tightly linked to their culture. In view of this, the South African government undertook the development of a National Recordal System (NRS). The NRS initiative aims to support the South African Department of Science and Technology (DST) in standardising the capturing, storing, management, maintenance, dissemination and protection of IKS in South Africa [4]. 
Recognizing that User Experience related challenges within IKS have largely been ignored in research, the objective of the research reported in this paper is to evaluate the User Experience (UX) challenges experienced by Indigenous Knowledge recorders in rural communities. The paper is structured as follows: Section 2 provides an overview of Indigenous Knowledge and its importance. Section 3 gives an overview of the current initiatives of developing Indigenous Knowledge Management System globally. Section 4 discusses related work in this field. The problem statement is identified in section5. The methodological aspects of this research are discussed in more detail in section 6 and the paper is concluded in Section 7.

\section{Overview of IK}

According to Ngulube in [5] and Ngozi in [6] there has been an increased interest in the significance of Indigenous Knowledge Systems (IKS) in sustainable development in developing countries around the world. Recent studies have shown that there is a need for people to contribute to Indigenous Knowledge Systems and to participate as both contributors and users of this knowledge [7]. In addition, a study done by Mondo in [8] reveal that Indigenous Knowledge Management has recently became a research focus area and can contribute to sustainable development. Therefore, it is very important for African communities to understand the importance of Indigenous knowledge and for the communities to play a major role in the collection/recording, safeguarding and dissemination of their own knowledge.

Indigenous Knowledge is considered to be unique, local and traditional knowledge to a specific community or society. IK is acquired through experiences and often kept in people's memories (mostly elderly people) and is conveyed to younger generations by telling stories, transferring skills and sharing experience [9] . IK is oral and transferred from generations through storytelling. In addition, Indigenous Knowledge plays an important role as it is viewed as an instrument that provides problem solving ideas and a way of surviving in rural communities and needs to be properly collected and protected [10].

From the descriptions of Indigenous Knowledge stated in the previous section, it can be concluded that a working understanding of Indigenous Knowledge is that it is a local knowledge which holds cultural practices of indigenous people and traditional to a specific community or society. It is a unique knowledge that has been gained from past events or experiences and often kept in people's memories (mostly elderly people) and is transferred orally from generations to generations [9],[3] and [11].

\subsection{Importance of IK}

Jangawe in [12] recognizes Indigenous Knowledge as a mechanism that has significance in the lives of most citizens especially in rural communities. Further he states that IK plays an important role in culture preservation, which then leads to the decrease in poverty. Also that IK is Scientific due to the fact that it is acquired through years and years of experience, thus it offers scientific clarification to issues and problems faced by the communities. Though IK adds value in the lives of the rural people, Scholars in the field agree to the fact that relying on elderly people's memories has challenges, since Indigenous Knowledge is available through the knowledge owner and is conveyed verbally, it is often not captured, documented or recorded electronically as elderly people pass on, the knowledge is lost and that has an impact in the economic development of the communities and the country in general [13].

Scholars have identified the reasons and importance of collecting. Recording of IK can prevent it from getting lost; protect it from unfair exploitation; conserve and promote it [9] 
and [3]. Tella in [11] describes the importance of IK and gives reasons why Indigenous knowledge should be recorded:

- IK provides problem solving strategies for local communities, especially for the poor;

- It represents an important contribution to global development knowledge;

- IK systems are at risk of becoming extinct;

- IK is relevant for the development process; and

- IK is an under-utilized resource in the development process.

In most IK-related studies, the people doing the IK collection within the villages are researchers in the field and academics. Furthermore, studies have indicated that collecting Indigenous Knowledge from the owners can be a problem in view of the fact that most IK owners will not want to share their knowledge with the people they do not know. The majority of IK owners feel that if they share or give out their knowledge, they will not have control over their IK.

Therefore to address the issue of trust, a study conducted by [14] reveals that in South Africa, the IK collection is done by members of the community. The person doing the interviewing and recording of IK is called the Indigenous Knowledge recorder (IK recorder). Usually, an IK recorder has the following characteristics: 1) Communicate well in a local language; 2) must be known or belong in the community; and 3) must be familiar with traditional culture and protocols within that particular community.

Indigenous Knowledge Management is comprised of a number of key elements namely capturing, storing, maintenance, dissemination and protection of Indigenous Knowledge and IK recording which allows the field workers to gather IK from within the communities using different methods. The author in [9] has discovered that there are different methods to collect and record IK including field workers visiting owners of the knowledge who are keen to share their knowledge, using recording devices such as video cameras, audio recorder, questionnaires and interviews.

The nominal IK collection process is seen from Sen in [15] as follows:

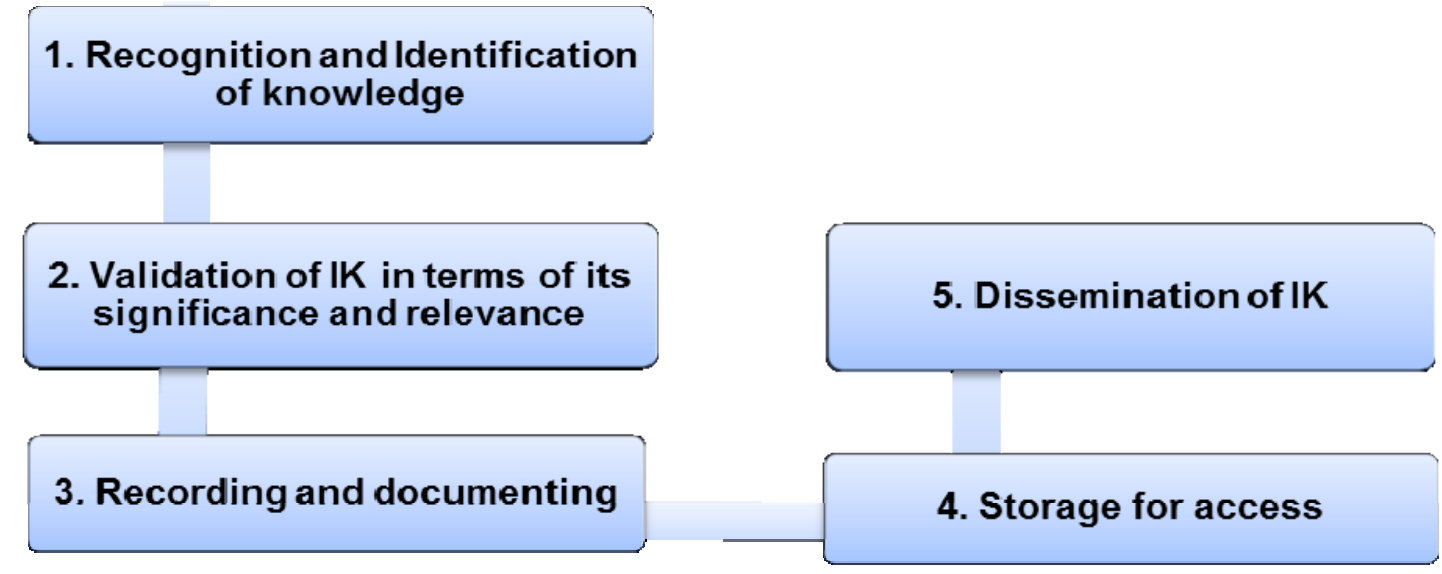

Figure1: IK collection process defined by Sen (2005)

The IK recorder encounters the system both as a process (3) and as a technological tool (4 and 5). In order to understand the issues that these IK recorders are encountering during their IK collection process or cataloguing (using the technology) this study will draw from the User Experience (UX) literature with the purpose of identifying key aspects of UX which this study should investigate. Necessarily, the next section provides a brief background to User Experience. 


\subsection{Understanding $U X$}

User Experience (UX) is defined as the experience that a person gains when he/she interacts with the product in terms of how easy or pleasing it is to user it. UX is about how a person feels after using a product or a system. [2] state that UX is dynamic due to the fact that as the circumstances changes it also changes. User Experience is recognised as an element that has an impact in the acceptance of the product (technology). Negative User Experience can have a negative effect in how people use the technology. Therefore it is very important for the development team (designers and developers) to understand the influence of user experience for a successful product and should put effort in investigation user experience in a product[16].

Therefore, this study aims to evaluate the User Experience (UX) challenges experienced by Indigenous Knowledge recorders in rural communities. It also evaluates the tools used to collect data as well as the processes defined to guide the IK recording/collection process. For the purpose of the research, the NRS project will be evaluated and the study will be conducted in three rural communities: one in Limpopo, one in Kwa-Zulu Natal and one North West provinces.

\section{Overview of IKS (current initiatives)}

There are quite a number of initiatives in several countries that have made an effort to implement Indigenous Knowledge Management Systems to assist in the collection, capturing and dissemination of Indigenous Knowledge [8]. As indicated earlier, the NRS project is another initiative undertaken by South African government. The South African government adopted a policy in 2004; to develop the Indigenous Knowledge Systems (IKS). The policy provided a platform for the recognition, affirmation, development, promotion and protection of IKS in South Africa. The NRS initiative aims to support the South African Department of Science and Technology (DST) in standardising the capturing, storing, management, maintenance, dissemination and protection of IKS in South Africa [14].

Some of the initiatives in other countries that have attempted to implement IKS are highlighted below:

\subsection{Biozulua database}

The Biozulua database initiative from Venezuela was established to record IK in order to encourage innovation using traditional knowledge and to facilitate the sharing of benefits between developers and the knowledge owners. During the collection, IK was collected and recorded from knowledge owners by researchers. Unfortunately, this initiative was stopped after the representatives of the indigenous communities from which IK was being sourced complained about their rights not being addressed by the system [17].

\subsection{The Traditional Knowledge Digital Library (TKDL)}

This initiative undertaken by India was developed to digitise and and translate traditional knowledge that was already published in existing texts in local languages in order to make it available for prior art searches [18].

\subsection{The Honey Bee Network}

The Honey Bee Network initiative is another initiative undertaken by India. In this project the fieldworkers who gather knowledge from communities are the students, teachers, and rural youth. The information is linked with the name of the traditional knowledge holder or community and their addresses. The traditional knowledge is collected in the format of 
unstructured stories from the traditional knowledge holders it is then translated into different languages, registered in a database, and published in a newsletter [19].

\subsection{Open source software tools for the protection, preservation and repatriation of IK - Australia}

The aim of these software tools is to enable indigenous communities to protect unique cultural knowledge and materials that have been preserved though digitisation. The focus of the system is the repatriation of physical indigenous knowledge artefacts held in museums and archives in digital format in the communities. Control over the stored IK is given to authorised members of the community in terms of the control of rights, accessibility and reusability of the digital resources, the enforcement of traditional laws, prevention of misuse of IK, and proper attribution to traditional owners [20].

\subsection{The China Traditional Chinese Medicine (TCM) patent database}

The Traditional Chinese Medicine (TCM) patent database was started in 2001 and is supported by the State Intellectual Property Office (SIPO) of China. Its aim is to cover the patents related to the TCM from April 1985 to date. The database provides 29 search entries with bibliographical data, subjects and applications/therapeutic effects. The patent database is deeply indexed and is reportedly updated monthly [21].

\section{Related work}

Fogwill in [22] presented a high level architecture of an Indigenous Knowledge System which included among its components the IK catalogue component. This component provided the technology support for the Indigenous Knowledge recording/collection process in South Africa which [9] evaluated.

A study presented by Alberts et al in [9] focused on the evaluation and refinement of the instruments used in communities. The evaluation took place in one community in Limpopo province, which was the pilot site of the project and during the study; the project was still in the refinement phase hence there was a need to perform the study.

This study builds on the work presented by Alberts et al in [9] by doing the similar study in more than one community. In this study, three communities in three provinces will be evaluated so as to generalize the list of UX challenges and in the end compare the results.

\section{Problem Statement}

As stated earlier, Indigenous knowledge in rural African communities is oral and is transferred through storytelling; the accuracy of such IK depends on the IK recorders who collect the knowledge from the IK owners. Hunter in [23] as well as Mondo in [8] indicate that since collection of IK is oral and often difficult to document, therefore audio visual digital tools have been implemented to facilitate the recording and documentation process, these includes digital video cameras, audio recorders, interactive tools and multimedia presentations. In South Africa, the NRS project has been launched and deployed in fourteen (14) communities in six (6) provinces of South Africa. However, there are still challenges with the quality of data collected in the field. It is then important to understand the UX issues and challenges encountered by the IK recorders (field workers) and which may have contributed to the poor quality of collected data. This makes the IK recording process and its supporting tools (technology and people) critical for the success of IKS in general. It is this appreciation of the role of IK recorders in the IKS that motivates the current study. 
Necessarily, this study focuses on the IK recording element which is the entry point of Indigenous Knowledge into the IKS. Therefore, understanding and resolving UX issues encountered by IK recorders at this level will help with the enhancement of the tools and processes that are used by the IK recorders to collect indigenous knowledge and that will improve the overall quality of data collected in the field.

\section{Methodology}

Hartely in [24] describes a case study as a research method that entails a detailed investigation. In a case study research, data is often collected over a period of time, within the context. Further, he states that the aim of a case study is to provide an analysis and indepth understanding of the context.

Yin in [25] defines a case study as "an empirical inquiry that investigates a contemporary phenomenon within its real-life context, especially when the boundaries between phenomenon and context are not clearly evident".

The following table depicts the characteristics of a case study and shows why it is suitable for the study.

Table 1: Characteristics of a case study (adapted from Yin 2009)

\begin{tabular}{|l|l|}
\hline Characteristics \\
\hline$>$ In-depth detailed data derived from wide data sources \\
$>$ Participant and nonparticipant observations \\
$>$ Non-interventionist \\
$>$ Empathic \\
$>$ Holistic treatment of phenomenon \\
$>$ What can be learned from a particular case
\end{tabular}

Considering the above, this study will adopt a Multiple Case study as a research strategy in order to investigate and get an in-depth understanding of the UX challenges experience by the IK recorders in rural communities and to understand the environment in which IK recorders operate and what issues do they come across when collecting Indigenous knowledge [25]. Furthermore, the South African initiative National Recordal System (NRS) will be used as a case study in three different communities and potentially different context. The purpose will be to explore one phenomenon in different situations.

\subsection{Data collection method}

Semi structured interviews and observations will be conducted to understand the reality of Indigenous Knowledge recorders in rural settings, how they collect data, and to understand the issues and challenges they encounter during the IK recording process. Participant observations will be used in order to evaluate and understand how the process that were defined and how they use the tools that were deployed in the field.

Structured interviews and questionnaires will also be used to seek data directly from the participants.

\section{Conclusions and Future Work}

The importance of the preservation of indigenous knowledge has rightly and appropriately been emphasized by proponents of IKS. However the preserved knowledge can only be useful if it is accurate. This makes the processes and tools used in collecting and storing this knowledge critically important as they directly impact the quality of the collected data (indigenous knowledge). The work in progress study reported in this paper is aimed at understanding the User Experience challenges and issues, which may arise and negatively 
affect the quality of the data collected through these processes and tools. To achieve this, multiple case study research strategy has been adopted. The User Experience related challenges are the main units of analysis in the multiple case studies.

The findings of the study will then be used to improve on the current IKS in South Africa, the National Recordal System (NRS), to capture and preserve the indigenous knowledge as accurately as possible. The future work entails finalizing the field data collection on User Experience challenges from the multiple case studies and to develop a framework for understanding these challenges. It is hoped that future publications will include the primary data and how these translate into technology requirements and process definitions for the collection of indigenous knowledge.

\section{References}

[1]. Rodil, K., et al., A new visualization approach to re-contextualize indigenous knowledge in rural Africa, in Human-Computer Interaction-INTERACT 20112011, Springer. p. 297-314.

[2]. Van Greunen, D. and A. Veldsman, A User-centric Way to Change Management for Rural Community Development, in Indigenous Knowledge Technology Conference2011. p. 134-135.

[3]. Subba Rao, S., Indigenous knowledge organization: An Indian scenario. International Journal of Information Management, 2006. 26(3): p. 224-233.

[4]. DST, Indigenous Knowledge Systems Policy, 2004, Department of Science and Technology: Pretoria, South Africa.

[5]. Ngulube, P., Managing and preserving indigenous knowledge in the knowledge management era: challenges and opportunities for information professionals. Information development, 2002. 18(2): p. 95-102.

[6]. Ngozi, B.O., African Indigenous Knowledge Systems (AIKS). Articulos, 2010. 7(2): p. 1-13.

[7]. Chikonzo, A., The potential of information and communication technologies in collecting, preserving and disseminating indigenous knowledge in Africa. The International Information \& Library Review, 2006. 38(3): p. 132-138.

[8]. Mondo, T.M., V. Baryamureeba, and D. Williams, Collaborative framework for supporting indigenous knowledge management, in ACM2007: Lisbon, Portugal. p. 163-170.

[9]. Alberts, R., G. Khalala, and O. Molefe, Collecting structured indigenous knowledge for enhanced exploration: the National Recordal System (NRS), South Africa. Knowledge Management for Development Journal, 2011. 7(3): p. 291-307.

[10]. Nhemachena, C., et al., Integrating indigenous knowledge systems (IKS) in improving rural accessibility and mobility (in support of the comprehensive rural development programme in South Africa). SATC 2011, 2011.

[11]. Tella, R.D., Towards promotion and dissemination of indigenous knowledge: a case of NIRD. The International Information \& Library Review, 2007. 39(3): p. 185-193.

[12]. Jangawe, M., Challenges and opportunities in the protection and preservation of Indigenous Knowledge in Africa. International Review of Information Ethics, 2007. 7: p. 1-8.

[13]. Kapuire, G.K. and E. Blake, An attempt to merge local and technological paradigms in the digital representation of indigenous knowledge. 2011.

[14]. Pretorius, R. and H. Bezuidenhout. National Recordal System IK holder catalogue process. in Indigenous Knowledge Technology Conference. 2011. Namibia.

[15]. Sen, B., Indigenous knowledge for development: Bringing research and practice together. The International Information \& Library Review, 2005. 37(4): p. 375-382.

[16]. Arhippainen, L. and M. Tähti. Empirical evaluation of user experience in two adaptive mobile application prototypes. in Proceedings of the 2nd international conference on mobile and ubiquitous multimedia. 2003.

[17]. Johnson, O., Venezuelan project establishes indigenous plant database. BMJ: British Medical Journal, 2002. 325(7357): p. 183.

[18]. Library, T.K.D. Traditional Knowledge Digital Library. 6 January 2014]; Available from: http://www.tkdl.res.in/tkdl/langdefault/common/Home.asp?GL=Eng.

[19]. Bee, H. Honey Bee Network. 6 January 2014]; Available from: http://www.sristi.org/hbnew/index.php.

[20]. Hunter, J., B. Koopman, and J. Sledge. Software tools for indigenous knowledge management. in Museums and the Web 2003. 2003.

[21]. WIPO. China Traditional Chinese Medicine Patent Database Search System. 6 January 2014]; Available from: iptk_bkk_09/wipo_iptk_bkk_09_topic5_2.pdf. 
[22]. Fogwill, T., et al. Software architecture for an indigenous knowledge management system. in Indigenous Knowledge Technology Conference (IKTC). 2011.

[23]. Hunter, J., The role of information technologies in indigenous knowledge management. Australian Academic and Research Libraries, 2005. 36(2): p. 113.

[24]. Hartley, J., Case study research. Essential guide to qualitative methods in organizational research, 2004: p. 323-333.

[25]. Yin, R.K., Case study research: Design and methods. Vol. 5. 2009: Sage. 